\title{
Review
}

\section{Effect of Transport Properties on Thermoelectric Generators Design, A review}

\author{
Meysam Karami Rad 1,* \\ 1 Department of Agricultural Machinery Engineering, Faculty of Agricultural Engineering and Technology, \\ University of Tehran, Karaj, Iran. \\ * Corresponding authors. 1- Tel: (+45) 50209780; Fax: (+45) 50209780; E-mail: mkaramirad@ut.ac.ir
}

\begin{abstract}
Due to the relatively low efficiency of thermoelectric (TE) generators, they have mostly been used in niche applications where the unique properties of TE generators outweigh their lack of efficiency, or conditions where it is more reasonable to base the optimal design on maximum generated power rather than maximum efficiency. The aim of this work is to investigate how the different fundamental transport properties (Seebeck coefficient, thermal conductivity and electrical resistivity) in materials with equal figure of merit $(Z T)$ impact TE module design and maximum power output. Further, we discuss and review the strategies to enhance material properties and the latest studies on the TE module performance. It is shown, that by increasing the power factor by a factor of 15 and decreasing the thermal conductivity by 13.33 in order to maintain ZT=1, power output is increased by $45 \%$. This effect is stronger in lower module fill factor and thermal resistance of the heat source and heat sink.
\end{abstract}

Keywords: Thermoelectric module; Seebeck coefficient; Figure of merit; Power factor; thermal conductivity; Contact resistance.

\section{Nomenclature}

\begin{tabular}{|c|c|c|c|}
\hline $\mathrm{PF}$ & Power Factor $10^{-3} \mathrm{~W} / \mathrm{K}^{2} \mathrm{~m}$ & $\mathrm{R}$ & Electrical resistance $[\Omega]$ \\
\hline$\kappa$ & Thermal conductivity $[\mathrm{W} / \mathrm{mK}]$ & $\mathrm{t}$ & Thickness \\
\hline$\rho$ & Electrical resistivity $[\Omega . m]$ & $\mathrm{V}$ & Voltage $[V]$ \\
\hline$\sigma$ & Electrical conductivity $[1 / \Omega . m]$ & I & Electrical current $[A]$ \\
\hline$S$ & Seebeck coefficient $[\mu V / K]$ & $Q$ & Heat flux $[W]$ \\
\hline$T$ & Temperature $[K]$ & $\mathrm{P}$ & Power $[W]$ \\
\hline$B i$ & Biot number & $n_{T C}$ & Number of thermocouples \\
\hline$h_{f}$ & Heat transfer coefficient $\left[\mathrm{W} / \mathrm{m}^{2} \mathrm{~K}\right]$ & & Abbreviation \\
\hline$l$ & Length $[m]$ & $\mathrm{COP}$ & Coefficient of Performance \\
\hline A & Surface Area $\left[\mathrm{m}^{2}\right]$ & DOS & Density of States \\
\hline$\dot{q}$ & Heat flux $\left[W / m^{2}\right]$ & FF & Fill Factor \\
\hline$j$ & Electrical current density $\left[\mathrm{A} / \mathrm{m}^{2}\right]$ & PGEC & $\begin{array}{c}\text { Phonon-Glass Electron- } \\
\text { Crystals }\end{array}$ \\
\hline A & Surface area $\left[\mathrm{m}^{2}\right]$ & TE & Thermoelectric \\
\hline
\end{tabular}




\begin{tabular}{|cc|cc|}
\hline & \multicolumn{3}{c|}{ Subscribes } \\
\hline C & Cold side & J & Junction \\
E & connection & leg & Thermoelectric leg \\
eff & Electrical & $\mathrm{M}$ & Module \\
F & Effective & $\mathrm{N}$ & n-type \\
$\mathrm{H}$ & Filler & $\mathrm{P}$ & P-type \\
$\mathrm{IC}$ & Hot side & $\mathrm{T}$ & Thermal \\
\hline
\end{tabular}

\section{Introduction}

Thermoelectric (TE) phenomena was introduced by Thomas Seebeck in 1821 and formulated by Onsager-Casimir methodology [1] in 1951 theoretically. Thermoelectric is the general name for Seebeck and Peltier effect with the same physical origin. Potentially, many energy conversion systems can be replaced by TE systems if the efficiency or coefficient of performance (COP) of TE systems becomes comparable to traditional technologies. For example, the conventional bismuth telluride, $B i_{2} \mathrm{Te}_{3}$, has the ZT of about 1 , whereas by ZT equal of $4.5, \mathrm{COP}$ of the commercial refrigerators can be achieved [2]. While TE materials and devices were well known in 1950s[3,4], there were no vision for ZT higher than one at that moment. ZT is a dimensionless number $\left(Z T=S \sigma^{2} T / \kappa\right)$, reflecting TE materialsquality, i.e., the higher ZT, the higher efficiency. In early 1990s, theoretical studies $[5,6]$ cleared that it is possible to improve the TE material properties by preparing them in quantum well superlattice structure and increae the figure of merit by factor of 13 potentially; However, Jian and Terry [7] in 2017 reviewed the paradigm-shifting mechanisms which kept TE researches trending in past few decades and counting 15 different mechanisms, concluded in past 60 years the maximum ZT value have doubled and in optimistic view have tripled. It does not sound impressive but they believe fourfold increase will significantly affect the renewable energy landscape. Since now, the low efficiency has restricted TE device applications for the specific applications $[8,9]$, including extreme environments - e.g. space exploration- waste heat rectory [10-12], micro generation for sensors [13], and wearable and flexible generators [14, 15]; albeit, advances in materials and devices has relatively broaden TE applications in past few decades [16]. There are the vast amount of literature on TE systems performance [17-25], interested readers referred to outstanding review papers [2, 25-29], but there are only a few studies describing the link between material transport properties and TE device performance [30-32]. Transport properties refers to fundamental macro scale properties including Seebeck coefficient $(S)$, electrical conductivity $(\sigma)$, and thermal conductivity $(\kappa)$. It is also common to report the ZT numerator called Power factor (PF) which reflects the electron related behavior of TE materials. It is the product of Seebeck coefficient square and electrical conductivity. Power factor and thermal conductivity do not affect TE device performance equally and ZT cannot reflect this inequality [33] and it is necessary to study the correlation between material properties and device level performance to provide the more clearer view both for scientist and engineers. LeBlanc [31] reviewed the effects of material properties on system design and argued that not only TE materials but also filler, substrates, solders and contacts, afterward manufacturing process, and cost are also important in TE device design process. She concluded that the most important issues in TE marketing are material selection, thermal and chemical stability, engineering of interfaces, interface materials, and optimization of hot and cold side thermal resistances. In another study [32], the material properties effects on three different kind of TE generators, e.g. a water heater, an automotive exhaust system, and an industrial furnace were studied and carbon nanotubes suggested as a 
promising material to overwhelm the interface contact challenges. The ultimate purpose of engineers is achieving the optimum design for the defined application. The optimization goal of energy conversion system is maximum efficiency. Design to achieve maximum power generation, disregarding the efficiency, is an optimization goal which it is reasonable in two conditions: the big enough heat sources and niche application with priorities other than cost, for example micro TE generators [34-38] for powering the wireless sensors. Such a studies have done for low temperature difference by neglecting the Thomson effect[34][39]. Base on author's survey, there is the lack of study which exactly comparing the effect of power factor and thermal conductivity on TE device performance in equal ZT condition. Prior to this, it is necessary to take a look on the current status of materials and each property ranges to provide the realistic estimation and suggestions.

Based on the above argument, it is necessary to have a bridge between the fundamental material properties and device performance parameters, which is only possible by having a picture of the whole TE generator device in mind and looking at the specific details simultaneously. To achieve this goal, the different strategies to enhance TE material properties will be discussed in Section 0 and their opportunities and challenges has been noted. Each property range and enhancement possibility has also been noted. In section 0 , different simulation approaches in field of thermoelectric are reviewed, and most important references were addressed to provide enough argument for selecting the simulation method and optimization procedure. Then the series of optimization process base on maximum power generation will perform with the balance between possible simplicity and validity in Section 4 and generated power for different combinations of materials properties, keeping ZT equal to one, has been investigated.

\section{Different TE Materials and the development strategies}

Since 1990s, after promising theoretical studies by physicists, predicting the nanostructuring potential to enhance TE material characteristics, the research on this topic has been increasing, mostly in material science and physics [2, 40-49]. Using desire material properties ranges for simulations is necessary to study the effect of material transport properties on device behavior, . Despite the number of outstanding reviews explaining the methods and strategies by detail, there was a lack of a database to provide data and evaluation possibilitis until 2013 when Gaultois et al. [48] reviewed more than 100 published papers and material properties were gathered in a free access online database [50] containing more than 18000 data points. Data was collected manually from digital publications using free software such as PlotDigitizer [51] and DataThief [52] at four temperatures $(300 \mathrm{~K}, 400 \mathrm{~K}, 700 \mathrm{~K}$ and $1000 \mathrm{~K})$. In some cases, they reported power factor instead of ZT because of the lack of thermal conductivity data and in some others numbers were interpolated or extrapolated to the desire temperatures.

A challenge to improve TE properties is controlling the electronic and thermal properties independently. It has successfully done in telluride materials for low-temperature applications However, this approach is not applicable at high temperatures [53]. To achieve this goal, there are four major ways, including sharp feature of the density of state (DOS) in bulk materials, sharp feature of DOS in low-dimensional materials [54], energy filtering and resonant levels to enhance the DOS [55, 56]. A more detailed categorized has been done by Jian and Terry in 2017 [7] that divided the development strategies into 15 categories, see Table 1, based on their origins. Furthermore, they gathered the latest and the most inspiring studies by more details. This paper uses the first category since it covers most of the strategies and enough details for defined purpose in this study.

Table 1- Summary of developing mechanism for thermoelectric materials extracted form [7].

\begin{tabular}{c|c}
\hline Origin & Mechanism \\
\hline
\end{tabular}




\begin{tabular}{c|c}
\hline \multirow{2}{*}{$\begin{array}{c}\text { Enabled by } \\
\text { defect }\end{array}$} & $\begin{array}{c}\text { Intrinsic point defects } \\
\text { Resonant levels } \\
\text { Band convergence } \\
\text { Topological defects } \\
\text { Enabled by } \\
\text { size effect }\end{array}$ \\
\hline Critical & Modulation doping \\
phenomena & Anharmonicity \\
& Rashba effect \\
& Topological states \\
& Spin Seebeck effect \\
\hline Other & The optimal carrier concentration \\
& Electron repository \\
& Multilocalization transport scenario \\
& Temperature-dependent mobility \\
& Band degeneracy and intervalley \\
& scattering \\
\hline
\end{tabular}

The difficulty to increase the TE material performance arises from the correlation between different parameters and the necessity to optimize all the parameter for the special working temperature. While the electrical conductivity and Seebeck coefficient depend on electron behavior, thermal conductivity only depends on electron and phonon, a quanta of crystal lattice vibration energy, behavior. Snyder and Toberer [40] studied the role of the electrons and phonons in thermal conductivity by using Wiedemann-Franz law, the relation between electron thermal and electrical conductivities [57]. They noted that if the ratio between phonon and electron part of thermal conductivity is very small, electron behavior is a dominating parameter and Seebeck coefficient determines ZT consequently. Tian et al. [55] reviewed the progresses in TE materials comprehensively and reported strategies to enhance the material performance including alloying in bulk materials by the idea of phonon-glass electron-crystals (PGEC) [58]; phonon rattling, putting the atom or molecules in void cage of the lattice structure, Skutterudites for temperature range between 500-900 K, Clathrates with low thermal conductivity, complex structures with low thermal conductivities, Half-heusler which are environment friendly and thermally stable, and having been focused on recently, Oxides, with advantage such as non-toxicity and thermal stability and suffering from low thermoelectric performances.

Nano-structuring is a way to enhance ZT in all kind of TE materials. It affects the TE behavior in two ways, quantum confinement by size reduction and phonon scattering by increasing boundaries and interfaces $[59,60]$. The nanostructure TE materials researches started by super lattices and quantum wells [61] and followed by bulk nanocomposites which are embedded nanoparticles or nanowires in a matrix or a hetero-structure. In [62] adding $\mathrm{Bi}$ nanoinclusions in nanostructured $B i_{2} \mathrm{Te}_{3}$ matrices was caused the reduction in lattice thermal conductivity and incensement in power factor by approximately doubling of the electrical conductivity and only a $20-25 \%$ reduction in Seebeck coefficient which is lead to $15-30 \%$ increase in power factor.

Skutterudites have $A B_{4} C_{12}$ chemical formula in which $\mathrm{A}$ is a rare earth; $\mathrm{B}$ is one of $\mathrm{Fe}, \mathrm{Co}, \mathrm{Ru}$ or $\mathrm{Os}$ and $C$ is one of $P, A s$ or $S b$ elements [63] and they know as the most promising mid temperature TE materials [64]. In a recent research[65], nano inclusions by reducing the grain size of the material was used to enhance the TE properties in bulk Skutterudites, $\mathrm{Yb}_{0.3} \mathrm{Co}_{4} \mathrm{Sb}_{12(1+x \%)}$ was synthesized, and the power factor increases dramatically to $4.710^{-3} \mathrm{~W} / K^{2} \mathrm{~m}$ due to energy filtering effect. Consequently, the $\mathrm{ZT}$ of $\approx 1.08$ has been achieved. In [66] $C u_{12-x} M_{x} S b_{13}$, where $\mathrm{M}$ is a metal was introduced with $\mathrm{ZT}$ and thermal conductivity near unit at $700 \mathrm{~K}$ and relatively lower cost which can be used in a wider variety of applications. 
Half-Heuslers have $A B X$ structure ( $A$ represents transition metal, noble metal or rare-earth element; $B$ represents transition metal or noble metal; and $\mathrm{C}$ is main group element) like cubic $M g A g A s$ that has one vacant sublattice [67]. The opportunities for tuning electronic and lattice make them a candidate for new high temperature materials, especially TE materials. Stability at high-temperature and low ZT of $p-$ type materials are challenges. Nano-compositing approach to enhance n-type $H f_{0.75} \mathrm{Zr}_{0.25} \mathrm{NiSn} n_{0.99} S b_{0.01}$ resulted $25 \%$ higher $\mathrm{ZT}$ ( $Z \mathrm{~T}=1, \mathrm{PF}=510^{-3} \mathrm{~W} / \mathrm{K}^{2} \mathrm{~m}$ and $\kappa=4.2 \mathrm{~W} / \mathrm{mK}$ at $600{ }^{\circ} \mathrm{C}$ ) than the previous methods [68], and heavy doping of p-type FeNbSb led to a $Z T \approx 1.5$ at $920^{\circ} \mathrm{C}[69]$. The outstanding review on Half-Heusler TE materials and their electron and phonon transport features can be found in [70].

The metal chalcogenides are composed of metal and chalcogen elements $(S, S e$ and $T e)$. In [71] the most recent strategies and development till 2016 in metal chalcogenides and their effect on TE enhancement mechanisms was presented. It introduces 237 references just for metal chalcogenides researches and also gives a comparison between different strategies. Difficult commercial production, poor mechanical behavior, low thermal stability, and toxicity are the most important problems which should be solve for this materials and nanostructures of shape, size, and interface and defect engineering are the most important and efficient ways to improve their behavior.

Oxides are another candidate for TE materials [72-74] although, they have poor intrinsic TE properties due to the low carrier mobility and high lattice thermal conductivity [7]. Materials like $\mathrm{BiCuSeO}$ are known as a good thermoelectric materials with ZT between 1.2 and 1.4 in $800-900 \mathrm{~K}$ temperature range[75], most of the improvement is because of intrinsic low thermal conductivity less than $1 \mathrm{~W} / \mathrm{mK}$ in recent years the discovered p-type $\mathrm{NaCo}_{2} \mathrm{O}_{4}$ showed the large Seebeck coefficients and low thermal conductivities $(<1$ $\mathrm{W} / \mathrm{mK}$ ) with $\mathrm{ZT}=1$ at $700-1000 \mathrm{~K}$ and the most promising candidates for $n$-type oxide thermoelectric materials include Perovskite-type $\mathrm{SrTiO}_{3}$ and $\mathrm{CaMnO}_{3}$ which by partially substituting $\mathrm{Dy}$ for $\mathrm{Sr}$, the thermal conductivity can drop to $3.4 \mathrm{~W} / \mathrm{mK}$ [55]. Another strategy to enhance the TE performance is increasing the Seebeck coefficient and electrical conductivity by increasing carrier mobility and suitable doping to find the material which can produce higher power even with lower efficiency. Oxides are relatively cheap and it might be reasonable to use them in large scale to produce electrical energy in case of waste heat recovery. Interestingly, decreasing fill factor increases the effect of better Seebeck coefficient that means by focusing on enhancing Seebeck coefficient instead of decreasing thermal conductivity it might generate higher energy with less material and costs. Nano-structuring can be considered as a way to improve the electron performance in Oxides [76]. By taking into account the above discussion and looking at the TE material properties online data base [50], the range transport properties to investigate their priority was chosen between $3 \times 10^{-3}$ and $1410^{-3} \mathrm{~W} / \mathrm{K}^{2} \mathrm{~m}$ for power factor, 1 and $5 \mathrm{~W} / \mathrm{mK}$ ) for thermal conductivity and 1 and $8 \Omega$. $\mathrm{m}$ for electrical resistivity.

\section{Theoretical approaches in Thermoelectric Studies}

Majority of theoretical models and simulations in thermoelectric phenomena describe the intrinsic TE behavior in materials and minority describes the best design for the TE generators and cooler with respect to material properties, application and working conditions. In context of linear non-equilibrium thermodynamics, the TE phenomena considered as the result of coupling between Seebeck, Peltier, Ohm, and Fourier phenomenological equations. The relation between Seebeck effect and Peltier effect described by generalized Onsager reciprocal relations[77]. Due to the shortcoming of classic methodologies to explain the thin layers materials behaviors, Ballistic-diffusive equations [78], the new heat transfer model derived from the Boltzmann equation, was used and the better experimental verification in comparison to Fourier and Cattaneo's equations has been observed. Machrafi et al. [79, 80], developed the size depended transport equations with respect to Knudson number to describe TE properties in nanofilms, nanocomposites and 
thin film nanocomposites. They indicated that extended irreversible thermodynamic (EIT) approach has a good agreement with experiments and more complex theories. A comprehensive review on continuum theories for TE materials and systems can be found in [77]. The overall overview on both continuum and fundamentals models since 2008 was provided in [81] [82][83]. Recently, Zebarjadi [84] studied the heat lost from sidewalls of TE legs and introduced a new modified Biot number $\left(B i=h_{f} l_{\text {leg }} / \kappa\right)$, to address the optimum sidewall insulation.

First-principles simulation is obtaining the intrinsic macroscopic properties, e.g. thermal, and electrical transport properties, of semiconductors with respect to their microscopic atomic structure [85]. In [86] both quantum and electron-phonon scattering used the non-equilibrium Green's function approach and claimed their predicated value for Seebeck coefficient in thin film materials show the similar trends with measured ones. By using the band theory to calculate the transport properties, the Seebeck coefficient can be defined as the average energy transported by the charge carriers. The simulation results show that the maximum power factor usually happens under highly doped condition and fine carrier concentration controlling in $H f Z r S n S b$ increase a $Z T$ value to 1.0 at $600-700^{\circ} \mathrm{C}$ [68]. Lemal et al. [87] proposed $\mathrm{Fe}_{2} Y Z$ semiconductor to obtain high power factor because of their single band orbitals, and introduced $\mathrm{Fe}_{2} \mathrm{NBGa}$ as the most promising $\mathrm{n}$ - type TE material with high power factor ranging from $5 \times 10^{-3}$ to $20 \times$ $10^{-3} \mathrm{~W} / K^{2} \mathrm{~m}$. Another first principal study [76] shows that in low temperatures, the thermal conductivity is proportional to $T^{3}$, because of weak phonon-phonon scattering and longer phonon MFPs while at high temperatures, thermal conductivity is proportional with $T^{-n}(\mathrm{n}=1-1.5)$ because of constant phonon specific heat at high temperature and linear increase of phonons with temperature. The first principal simulation is a strong approach to predict the material properties, and explains the mechanism and define the limitations and boundaries for advancement [88].

The simulation approach should be chosen according to simulation purpose that is investigation of the transport properties effect on TE device performances parameter. Authors focus is on general TE devices where it is not necessary to use specific approaches for thin layers. Continuum Onsger-Casimier relationships can provide sufficient information to support this study. Moreover, the assumptions, such as one dimensional heat transfer and neglecting Thomson effect, do not affect the simulations negatively since this study aims to find that which transport properties has more intense effect on maximum power generation.

\section{Calculations}

In order to investigate the effect of the different material properties on the module design parameters, methodology similar to [34-38] is used with some modifications. Figure 1 depicts the thermal and electrical models for thermoelectric generators (TEG). The TEGs model is a coupled model between electrical and thermal fields which interact by Ohm's law, Seebeck voltage and Thomson heat. 

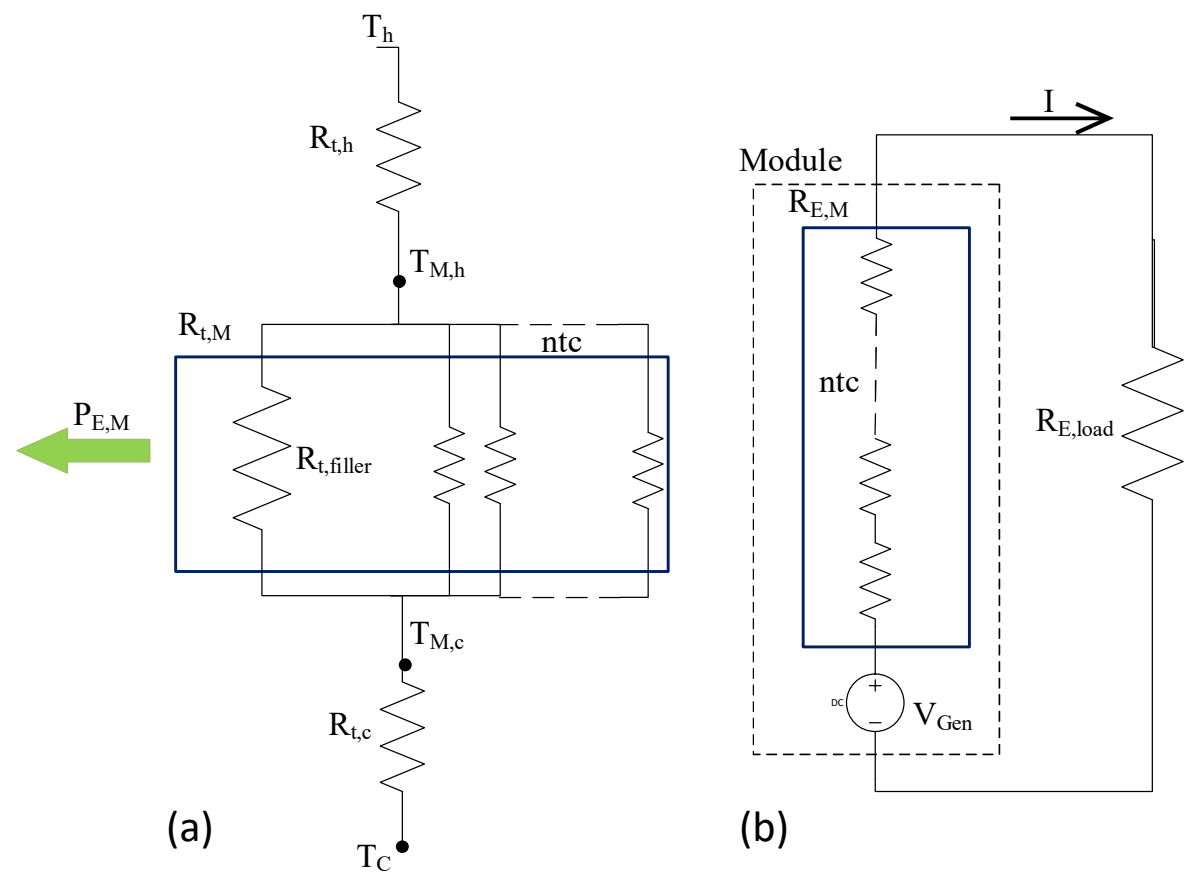

(b)

Figure 1- (a) Thermal resistance in one dimensional model, (b) the equivalent electrical model for thermoelectric generator.

To calculate thermal and electrical resistances of the module in the pre-process level of simulations the temperature independent electrical resistance is:

$$
R_{E . p / n}=\rho_{p / n} \frac{l_{\text {leg }}}{A_{p / n}}
$$

The electrical resistances of all interconnections material are calculated geometrically:

$$
R_{E . I C}=2 n_{T C} \rho_{I C} \frac{1+\sqrt{\frac{1}{F F}}}{t_{I C}}
$$

The contact resistance of module can be defined as:

$$
R_{E . \text { con }}=n_{T C}\left(\frac{\rho_{\text {con }}}{A_{n}}+\frac{\rho_{\text {con }}}{A_{p}}\right)
$$

Therefore, the total electrical resistance of the module is:

$$
R_{E . M}=n_{T C}\left(R_{E . n}+R_{E . p}\right)+R_{E . I C}+R_{E . c o n}
$$

The thermal resistances are calculated by similar method for each elements and part of the module as

$$
R_{t . p / n}=\frac{l_{\text {leg }}}{\kappa_{p / n} A_{p / n}}
$$

For the filler substrate and passive layers, we get:

$$
\begin{gathered}
R_{t . F}=\frac{l_{\text {leg }}}{\kappa_{F} A(1-F F)}(\mathrm{a}) \\
R_{t . \text { sub }}=\frac{l_{\text {sub }}}{\kappa_{\text {sub }} A}
\end{gathered}
$$




$$
R_{t . p a s s}=\frac{l_{\text {pass }}}{\kappa_{\text {pass }} A}(\mathrm{c})
$$

where $\kappa_{\text {sub }}, \kappa_{\text {pas }}, l_{\text {sub }}$ and $l_{\text {pass }}$ are substrate and passive layers thermal conductivity and thickness, respectively. The thermal resistance of the module. In the module, the filler and thermocouples are parallel thermally while they are electrically in series with interconnections and contact resistances which it means:

$$
\begin{aligned}
R_{t . M}=R_{t . s u b}+ & R_{t . p a s s} \\
& +\left(\frac{n_{T C}}{R_{t, p}}+\frac{n_{T C}}{R_{t, n}}+\frac{1}{R_{t, F}}\right)^{-1}
\end{aligned}
$$

In order to provide the maximum power, according to simplified model in [89] the electrical load should be equal to effective inner resistance $\left(R_{E . L o a d}=R_{E, M}^{e f f}\right)$ :

$$
R_{E, M}^{e f f}=R_{E . M}+T_{c} n_{T C}^{2}\left(S_{p}-S_{n}\right)^{2}\left(R_{t, c}-R_{t, h}\right) \frac{R_{t, M}}{R_{t, M}+R_{t, c}+R_{t, h}}
$$

After defining related variable and introducing them into the system on nonlinear equations for TEG module, the system of equations solved numerically in MATLAB. The governing equations for TE module based on energy balance equations and Seebeck law can be derived with respect to Figure 1:

$$
\begin{aligned}
& I=\frac{S_{M} \Delta T_{M}}{R_{E, M}+R_{E, \text { load }}} \\
& \frac{T_{h}-T_{M, h}}{R_{t, h}}=\frac{T_{M, c}-T_{c}}{R_{t, c}}+I^{2} R_{E, \text { load }} \\
& \frac{T_{h}-T_{M, h}}{R_{t, h}}=\frac{\Delta T_{M}}{R_{t, M}}+I S_{M} T_{M, h}-I^{2} \frac{R_{E, \text { load }}}{2}
\end{aligned}
$$

where $S_{M}=n_{T C}\left(S_{p}-S_{n}\right)$ is the Seebeck coefficient of the module. The final simulation step is the postprocessing to define meaningful parameters. Table 2 shows the temperature independent material properties which belong to common TE materials. The figure of merit for all materials kept one in all simulations. All the reported values for power in this study are based on maximum power optimization. Figure 2 shows the flowchart of simulation process to find the maximum power for the different designs. 


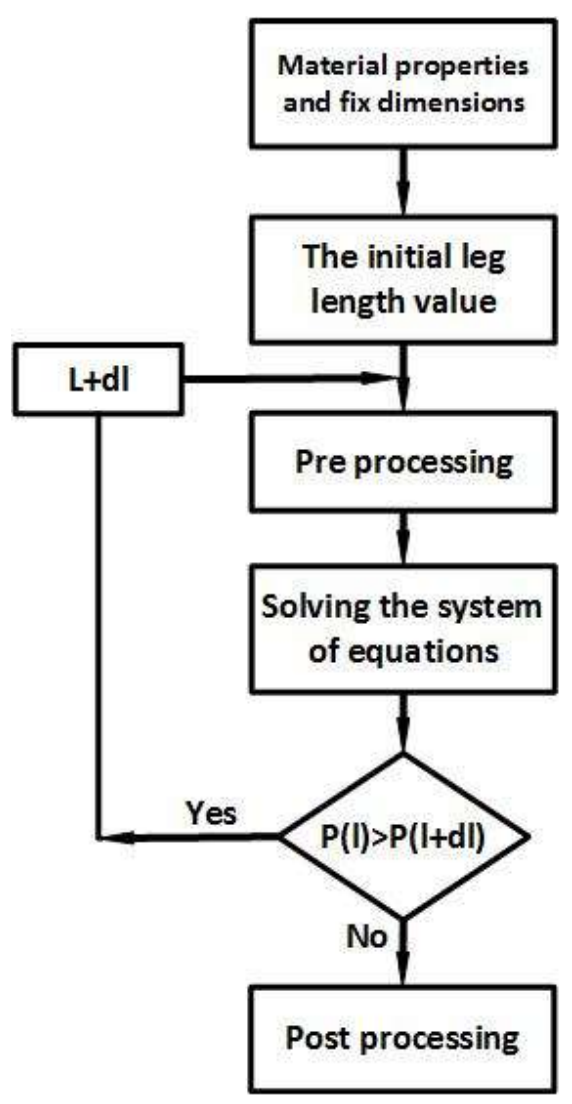

Figure 2-flowchart of all process including finding the optimized leg length.

Table 2-Initial TE module parameters in simulations.

\begin{tabular}{cc||cc}
\hline \hline$T_{h}(K)$ & 312.15 & $l_{\text {pass }}(\mathrm{nm})$ & 100 \\
$T_{c}(K)$ & 302.15 & $\kappa_{\text {pass }}(\mathrm{W} / \mathrm{mK})$ & 29 \\
$A\left(\mathrm{~cm}^{2}\right)$ & 1 & $t_{I C}(\mu m)$ & 20 \\
$n_{T C}$ & 100 & $\rho_{I C}(\Omega . m)$ & $1.68 \times 10^{-8}$ \\
$\kappa_{F}(W / m K)$ & 0.024 & $\rho_{I C}\left(\Omega . m^{2}\right)$ & $5 \times 10^{-11}$ \\
$L_{\text {sub }}(\mu m)$ & 200 & $R_{t, h}(W / K)$ & 1 \\
$\kappa_{\text {sub }}(W / m K)$ & 25 & $S(\mu V / K)$ & 173 \\
$\kappa_{\text {leg }}(W / m K)$ & 0.9 & $P F($ & 3 \\
& & $\left.\times 10^{-3} W / K^{2} m\right)$ & \\
\hline
\end{tabular}

The output power for each set of simulation was been normalized based on the specific base condition. To calculate the absolute value for the power, normalized power $\left(\mathrm{P} / \mathrm{P}_{0}\right)$ should be multiplied in the corresponded initial condition $\left(P_{0}\right)$.

\section{Results and Discussion}

The effect of different material properties on maximum possible electrical power was investigated. For this purpose, it was assumed the $n$ - and $p$-type materials have identical thermoelectric properties, with same cross sectional area. To evaluate the effect of power factor and thermal conductivity several set of the simulations were performed. In each set, different combinations of the Seebeck coefficient and thermal conductivity were used to provide constant figure of merit $(Z T=1)$. In other words, the higher power factor 
leads to the higher thermal conductivity to keep ZT constant. In thermoelectric module design there are many correlated parameters which affect the module performance. To deal with this complexity, the matched power was normalized based on the base condition, mentioned in the caption of each figures.

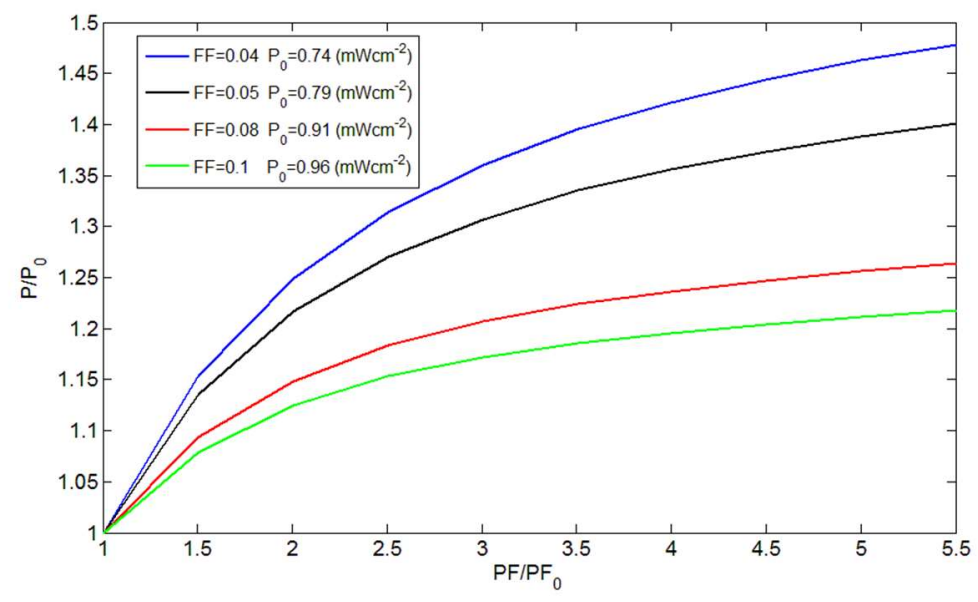

Figure 3- Normalized maximum power according to normalized power factor for different conditions with the different fill factor $\left(\mathrm{T}_{\mathrm{c}}=302.15 \mathrm{~K}, \mathrm{~T}_{\mathrm{h}}=312.15 \mathrm{~K}, \mathrm{PF}_{\mathrm{o}}=3 \times 10^{-3} \mathrm{~W} / \mathrm{K}^{2} \mathrm{~m}\right.$ and $\left.\mathrm{R}_{\mathrm{t}, \mathrm{c}}=10 \mathrm{~W} / \mathrm{Km}\right)$ ).

Figure 3 shows the normalized maximum power according to normalized power factor. It is clear that materials with higher power factor can produce higher amount of electrical power. From $P F / P F_{0}=1$ to 5.5 ( $P F$ vary from 3 to $15 \times 10^{-3} \mathrm{~W} / \mathrm{K}^{2} \mathrm{~m}$ ) and thermal conductivity from 1 to $5 \mathrm{~W} / \mathrm{Km}$, consequently), according to literature, most of the studied material with high ZT are in this domain [50]. The normalized power increases steeply by increasing normalized power factor from 1 to 5.5, then it relatively leveled out at specific power factor. The higher generated power in higher power factor is due to higher thermal conductivity that means an increment in transferred heat and, consequently, higher electrical power even with lower efficiency. In current TEG applications, e.g. wireless sensors and waste heat recovery, the heat source is large enough to provide enough energy and the problem is providing higher electrical power. Therefore, it is acceptable to produce enough energy even if it costs lower conversion efficiency. An example for material which has high power factor and thermal conductivity are Half-Heusler materials which by alloying and nanostructuring give (ZT=1) in p-type $Z r_{1-x} T i_{x} C o S b_{0.8} S n_{0.2}$ and n-type $H f_{1-x} Z r_{x} N i S b_{0.99} S n_{0.01}$ [90]. The critical value for power factor depends on the electrical contact and interconnection resistance, although $15 \times 10^{-3} \mathrm{~W} / \mathrm{K}^{2} \mathrm{~m}$ can be a rough estimation for suitable power factor for materials which have the $Z T=1$, since the used values for contact resistance and interconnections in this study are the most convenient values. The effect of fill factor on the power factor is clearly visible in Figure 3. Decreasing the fill factor from 0.1 to 0.04 increases the effect of power factor from $21 \%$ to $48 \%$, means well designed modules less amount of TE materials in $\mathrm{FF}=0.04\left(1.1 \mathrm{~mW} / \mathrm{cm}^{2}\right)$ can produce the same amount of energy with the $\mathrm{FF}=0.1\left(1.16 \mathrm{~mW} / \mathrm{cm}^{2}\right)$. 


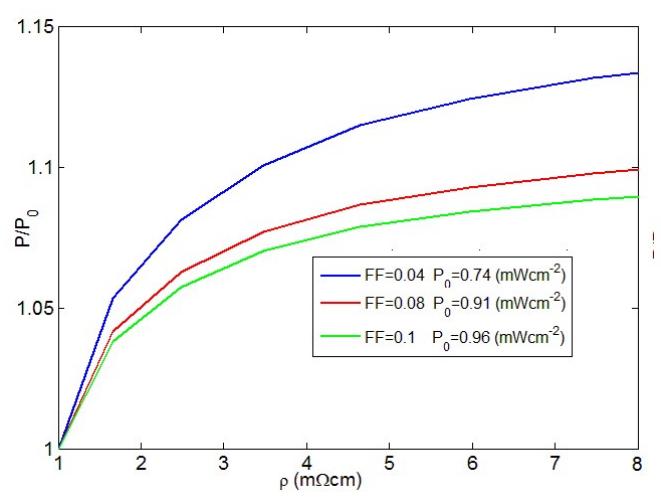

Figure 4- Normalized maximum power according to material electrical resistance with the different Fill factor $\left(\mathrm{T}_{\mathrm{c}}=302.15 \mathrm{~K}, \mathrm{~T}_{\mathrm{h}}=312.15 \mathrm{~K}, \mathrm{FF}=0.1\right.$ and $\left.\mathrm{R}_{\mathrm{t}, \mathrm{c}}=10 \mathrm{~K} / \mathrm{W}\right)$.

The second scenario is the materials with same thermal conductivity but different electrical conductivities. As shown in Figure 4, increasing the electrical resistivity increases Seebeck coefficient in equal PF that means the higher power. However, the Joule heating increases and it suppresses the power increase.

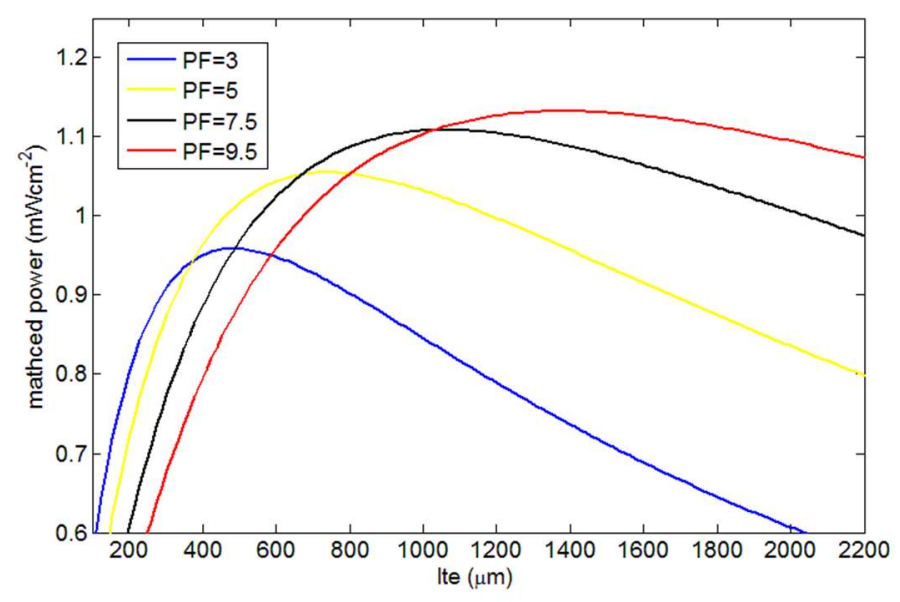

Figure 5- Optimal leg length for different power factor $(\mathrm{FF}=0.1)$.

The matched power for different leg length and power factor is presented in Figure 5. Increasing the power factor causes the higher matched power in higher leg length. The higher leg length is needed to provide the proper temperature difference in higher thermal conductivity. One way to enhance power and efficiency is lowering the leg length to decrease the thermal impedance. However, this approach would cause some practical problems like cracks and degradation[30]. Kim et al, [91] proposed a methodology to deal with $T E$ performance and device reliability. In a case study for p-type $B i_{2} \mathrm{Te}_{3}$ they claimed it is not suitable to enhance $Z T$ by reducing the thermal conductivity less than $0.5 \mathrm{~W} / \mathrm{mK}$. This is a different view compare to view in this study, however both of them refer that there should be a limitation for lowering thermal conductivity. Summary of different models aiming to predict the TE performance in large temperature difference and defining minimum acceptable thermal conductivity value can be found in [30].

Figure 6 shows effect of variation of material properties on figure of merit of the module. The all module and system level parasitic effects on TE generators are translated to dimensionless module figure 
of merit. Figure 6 indicates the choice of material can decrease the module figure of merit from 0.9 to 0.7 that corresponds to have the material with $Z T=1.3$ instead of 1 that is a significant improvement in the material level. Figure 7 (a) and (b) show the effect of heat source and sink thermal resistances. In case that the optimization is based on the power generation, the lower thermal resistance in heat source and sinks increases the power generated by increasing the transferred heat across the module. Figure 7 shows that by reduction of the heat sink thermal resistance from 10 to one the matched power can increase from 1.1 to $5.46 \mathrm{~mW} / \mathrm{cm}^{2}$. However reduction of heat sink thermal resistance is the critical technical problem and cannot be achieved simply. The results can help choose the proper material for each $n$ - and p-type elements. Regarding to material selection, it should be noted that there are more consideration like operating temperature and toxicity which should be took into account.

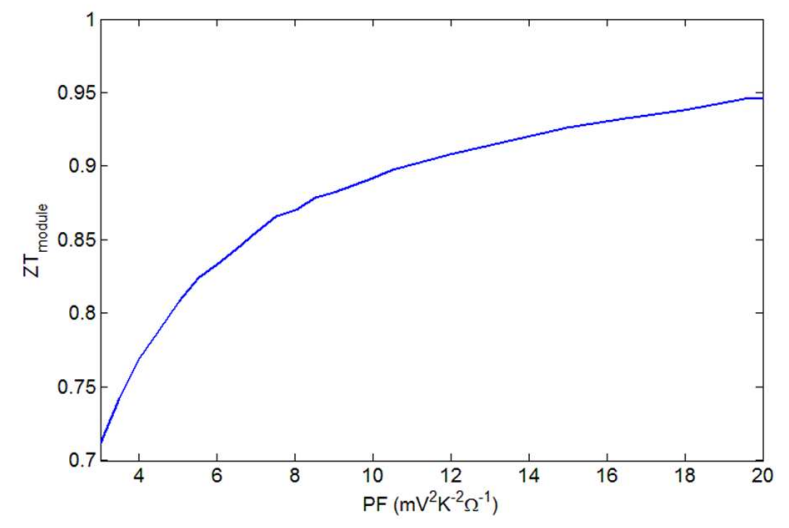

Figure 6- Module figure of merit for different power factor ( $\mathrm{FF}=0.1)$.

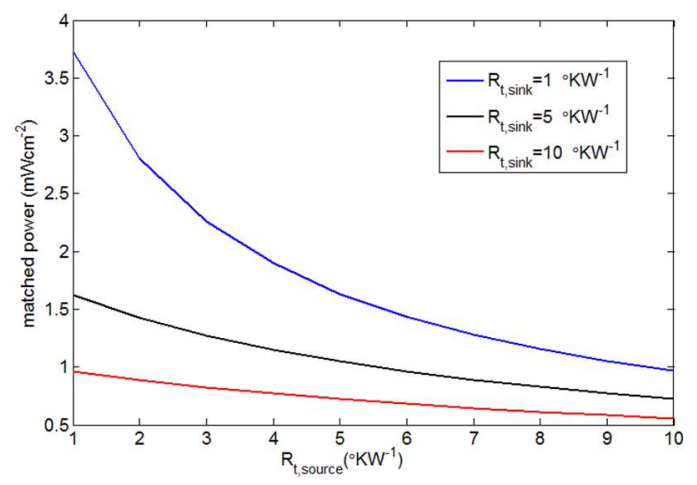

(a)

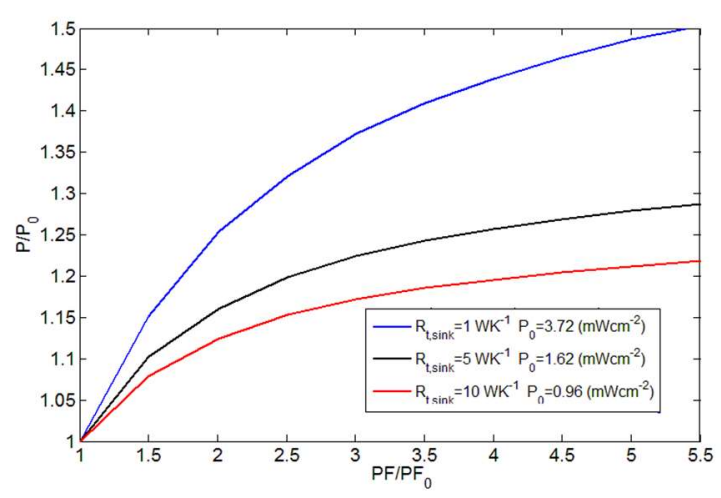

(b)

Figure 7- (a) Effect of source and sink thermal resistance. (b) Effect of power factor in different heat sink thermal resistance $\mathrm{T}_{\mathrm{c}}=302.15 \mathrm{~K}, \mathrm{~T}_{\mathrm{h}}=312.15 \mathrm{~K}$ and $\mathrm{FF}=0.1$ ).

\section{Conclusion}

In this work, the effect of the materials transport properties on TE systems performance was studied. TEGs are mostly used in a place which there is no other alternatives, like micro power generators for wireless sensors, heat recovery and space explorations which the heat sources are much bigger than the energy demand and it is reasonable to design the TE system to reach enough power instead of the higher efficiency. The most important approaches to enhance TE material performance were addressed. The TE module design is an interdisciplinary subject across physics, chemistry and materials science and 
engineering, in which intrinsic material parameters and device performance interact and requires general view during the design process. For example, the fill factor, and thermal resistance of the heat source/sink can strongly affect the optimum required power factor. According to the simulation results, the power factor has a significant effect on TE performance. Enhancement of the ZT by increasing power factor is the better enhancement strategy compared with decreasing the thermal conductivity. The importance of power factor should not be disregarded by focusing on ZT. This conclusion is furthermore supported by other recent studies with different arguments in [92] and [30], that address the thermos-mechanical durability and stability problems caused by low thermal conductivity.

Among current TE materials categories, oxides and Skutterudites show better electron performance by nanostructuring and doping control and according to simulation results, they are more promising. Because of intrinsic low thermal conductivity $(\sim 2 \mathrm{~W} / \mathrm{mK})$ and power Factor $\left(\sim 0.1-1 \times 10^{-3} \mathrm{~W} / \mathrm{K}^{2} \mathrm{~m}\right)$ in Oxides, there is a potential to modify electron mobility and carrier concentration to enhance their power factor; even though, it costs higher thermal conductivities [93]. A comprehensive review on power factor enhancement strategies [94] shows there is a long path to enhance the power factor, and many opportunities to failure and success. In recent years, Carbon nanotubes [95, 96], organic [97, 98], and inorganic-organic[99] materials have shown great potential to enhance thermoelectric power factors, For instance, new carbon nanotubes films and fibers with power factor of $2.48 \times 10^{-3} \mathrm{~W} / \mathrm{K}^{2} \mathrm{~m}$ at room temperature [95], new organic multidimensional conjugated nanomaterials $\left(2.71 \times 10^{-3} \mathrm{~W} / \mathrm{K}^{2} \mathrm{~m}\right)$ [98], and flexible inorganic-organic superlattice $\left(0.904 \times 10^{-3} \mathrm{~W} / \mathrm{K}^{2} \mathrm{~m}\right)$ [99] are some of candidates. Finally, it is worthy to note that progress in this field has not been fast as much as expected at the beginning of 1990s, nevertheless progress in material development and selection of optimization strategy leaves a lot of opportunities for future studies.

\section{Acknowledgements}

Authors would like to acknowledge the research center of the University of Tehran, Iran, and NanoCaTe project granted by $7^{\text {th }}$ Framework Programme under Grant No. 604647 for financial support of this work.

\section{References}

1. [1] De Groot SR, De Groot SR. Thermodynamics of irreversible processes: North-Holland Amsterdam; 1951.

2. [2] Twaha S, Zhu J, Yan Y, Li B. A comprehensive review of thermoelectric technology: Materials, applications, modelling and performance improvement. Renewable and Sustainable Energy Reviews. 2016;65:698-726.

3. [3] Telkes M. The Efficiency of Thermoelectric Generators. I. Journal of Applied Physics. 1947;18:1116-27.

4. [4] Goldsmid HJ. Introduction to thermoelectricity: Springer; 2009.

5. [5] Hicks L, Dresselhaus M. Effect of quantum-well structures on the thermoelectric figure of merit. Physical Review B. 1993;47:12727.

6. [6] Hicks L, Dresselhaus M. Thermoelectric figure of merit of a one-dimensional conductor. Physical review B. 1993;47:16631.

7. [7] He J, Tritt TM. Advances in thermoelectric materials research: Looking back and moving forward. Science. 2017;357:eaak9997.

8. [8] Champier D. Thermoelectric generators: A review of applications. Energy Conversion and Management. 2017;140:167-81.

9. [9] Kane A, Verma V, Singh B. Optimization of thermoelectric cooling technology for an active cooling of photovoltaic panel. Renewable and Sustainable Energy Reviews. 2017;75:1295-305.

10. [10] Ovik R, Long B, Barma M, Riaz M, Sabri M, Said S, et al. A review on nanostructures of high-temperature thermoelectric materials for waste heat recovery. Renewable and Sustainable Energy Reviews. 2016;64:63559. 
11. [11] Temizer İ, İlkılıç C. The performance and analysis of the thermoelectric generator system used in diesel engines. Renewable and Sustainable Energy Reviews. 2016;63:141-51.

12. [12] Guo L, Lu Q. Potentials of piezoelectric and thermoelectric technologies for harvesting energy from pavements. Renewable and Sustainable Energy Reviews. 2017;72:761-73.

13. [13] Akhtar F, Rehmani MH. Energy replenishment using renewable and traditional energy resources for sustainable wireless sensor networks: A review. Renewable and Sustainable Energy Reviews. 2015;45:769-84.

14. [14] Siddique ARM, Mahmud S, Van Heyst B. A review of the state of the science on wearable thermoelectric power generators (TEGs) and their existing challenges. Renewable and Sustainable Energy Reviews. 2017;73:730-44.

15. [15] Choi J, Cho K, Kim S. Flexible Thermoelectric Generators Composed of n-and p-Type Silicon Nanowires Fabricated by Top-Down Method. Advanced Energy Materials. 2017;7.

16. [16] Zhao D, Tan G. A review of thermoelectric cooling: materials, modeling and applications. Applied Thermal Engineering. 2014;66:15-24.

17. [17] Gurevich YG, Logvinov G. Theory of thermoelectric cooling in semiconductor structures. Revista mexicana de física. 2007;53:337-49.

18. [18] Date A, Date A, Tan L, Akbarzadeh A. THEORETICAL ANALYSIS TO DETERMINE THE SOLAR CONCENTRATION LIMIT WITH PASSIVE COOLING OF SOLAR CELLS. target.224:4.

19. [19] Yazawa K, Shakouri A. System optimiztion of hot water concentrated solar thermoelectric generation. Thermal Issues in Emerging Technologies Theory and Applications (ThETA), 2010 3rd International Conference on: IEEE; 2010. p. 283-90.

20. [20] Lertsatitthanakorn C, Khasee N, Atthajariyakul S, Soponronnarit S, Therdyothin A, Suzuki RO. Performance analysis of a double-pass thermoelectric solar air collector. Solar Energy Materials and Solar Cells. 2008;92:1105-9.

21. [21] Caballero GEC, Castellanos LSM, Cobas VRM, Lora EES. MODELING OF A CAVITY RECEIVER OF A SOLAR DISH/STIRLING SYSTEM. 2013.

22. [22] Baranowski LL, Snyder GJ, Toberer ES. Concentrated solar thermoelectric generators. Energy \& Environmental Science. 2012;5:9055-67.

23. [23] Chen G. Theoretical efficiency of solar thermoelectric energy generators. Journal of Applied Physics. 2011;109:104908.

24. [24] Kraemer D, McEnaney K, Chiesa M, Chen G. Modeling and optimization of solar thermoelectric generators for terrestrial applications. Solar Energy. 2012;86:1338-50.

25. [25] Sajid M, Hassan I, Rahman A. An overview of cooling of thermoelectric devices. Renewable and Sustainable Energy Reviews. 2017;78:15-22.

26. [26] Gao HB, Huang GH, Li HJ, Qu ZG, Zhang YJ. Development of stove-powered thermoelectric generators: A review. Applied Thermal Engineering. 2016;96:297-310.

27. [27] Najjar YS, Kseibi MM. Thermoelectric stoves for poor deprived regions-A review. Renewable and Sustainable Energy Reviews. 2017;80:597-602.

28. [28] Ding L, Akbarzadeh A, Tan L. A review of power generation with thermoelectric system and its alternative with solar ponds. Renewable and Sustainable Energy Reviews. 2018;81:799-812.

29. [29] Huen P, Daoud WA. Advances in hybrid solar photovoltaic and thermoelectric generators. Renewable and Sustainable Energy Reviews. 2017;72:1295-302.

30. [30] Kim HS, Liu W, Ren Z. The bridge between the materials and devices of thermoelectric power generators. Energy \& Environmental Science. 2017.

31. [31] LeBlanc S. Thermoelectric generators: Linking material properties and systems engineering for waste heat recovery applications. Sustainable Materials and Technologies. 2014;1:26-35.

32. [32] LeBlanc S, Goodson KE. System and Material Parameter Effects on Thermoelectric Power Generation in Three Combustion Systems. energy.6:7.

33. [33] Dunham MT, Barako MT, LeBlanc S, Asheghi-Roudheni M, Chen B, Goodson K. Modeling and Optimization of Small Thermoelectric Generators for Low-Power Electronics. ASME 2013 International 
Technical Conference and Exhibition on Packaging and Integration of Electronic and Photonic Microsystems: American Society of Mechanical Engineers; 2013. p. V001T03A9-VT03A9.

34. [34] Dunham MT, Barako MT, LeBlanc S, Asheghi M, Chen B, Goodson KE. Power density optimization for micro thermoelectric generators. Energy. 2015;93:2006-17.

35. [35] Yazawa K, Shakouri A. Optimization of power and efficiency of thermoelectric devices with asymmetric thermal contacts. Journal of Applied Physics. 2012;111:024509.

36. [36] Apertet Y, Ouerdane H, Glavatskaya O, Goupil C, Lecoeur P. Optimal working conditions for thermoelectric generators with realistic thermal coupling. EPL (Europhysics Letters). 2012;97:28001.

37. [37] Strasser M, Aigner R, Franosch M, Wachutka G. Miniaturized thermoelectric generators based on polySi and poly-SiGe surface micromachining. Sensors and Actuators A: Physical. 2002;97:535-42.

38. [38] Glatz W, Muntwyler S, Hierold C. Optimization and fabrication of thick flexible polymer based micro thermoelectric generator. Sensors and Actuators A: Physical. 2006;132:337-45.

39. [39] Rezania A, Rosendahl L, Yin H. Parametric optimization of thermoelectric elements footprint for maximum power generation. Journal of Power Sources. 2014;255:151-6.

40. [40] Snyder GJ, Toberer ES. Complex thermoelectric materials. Nature materials. 2008;7:105-14.

41. [41] Koumoto K, Terasaki I, Funahashi R. Complex oxide materials for potential thermoelectric applications. Mrs Bulletin. 2006;31:206-10.

42. [42] Kamarudin MA, Sahamir SR, Datta RS, Long BD, Mohd Sabri MF, Mohd Said S. A Review on the Fabrication of Polymer-Based Thermoelectric Materials and Fabrication Methods. The Scientific World Journal. 2013;2013.

43. [43] LeBlanc S, Yee SK, Scullin ML, Dames C, Goodson KE. Material and manufacturing cost considerations for thermoelectrics. Renewable and Sustainable Energy Reviews. 2014;32:313-27.

44. [44] Sundarraj P, Maity D, Roy SS, Taylor RA. Recent advances in thermoelectric materials and solar thermoelectric generators - a critical review. RSC Advances. 2014;4:46860-74.

45. [45] Liu W, Yan X, Chen G, Ren Z. Recent advances in thermoelectric nanocomposites. Nano Energy. 2012;1:42-56.

46. [46] Tian Z, Lee S, Chen G. Heat Transfer in Thermoelectric Materials and Devices. Journal of Heat Transfer. 2013;135:061605.

47. [47] Medlin D, Snyder G. Interfaces in bulk thermoelectric materials: a review for current opinion in colloid and interface science. Current Opinion in Colloid \& Interface Science. 2009;14:226-35.

48. [48] Gaultois MW, Sparks TD, Borg CK, Seshadri R, Bonificio WD, Clarke DR. Data-driven review of thermoelectric materials: performance and resource considerations. Chemistry of Materials. 2013;25:2911-20.

49. [49] Ortega S, Ibáñez M, Liu Y, Zhang Y, Kovalenko MV, Cadavid D, et al. Bottom-up engineering of thermoelectric nanomaterials and devices from solution-processed nanoparticle building blocks. Chemical Society Reviews. 2017.

50. [50] Energy material data mining. university of Santa Barbara; 2013.

51. [51] Huwaldt JA. Plot digitizer. 2013.

52. [52] Tummers B. DataThief III. 2006. URL http://datathief org. 2016.

53. [53] Brinks P, Kuiper B, Breckenfeld E, Koster G, Martin LW, Rijnders G, et al. Enhanced thermoelectric power factor of $\mathrm{NaxCoO} 2$ thin films by structural engineering. Advanced energy materials. 2014;4.

54. [54] Heremans JP, Jovovic V, Toberer ES, Saramat A, Kurosaki K, Charoenphakdee A, et al. Enhancement of thermoelectric efficiency in PbTe by distortion of the electronic density of states. Science. 2008;321:554-7.

55. [55] Tian Z, Lee S, Chen G. A comprehensive review of heat transfer in thermoelectric materials and devices. Ann Rev Heat Transfer. 2014;17:425-83.

56. [56] Zhang Q, Wang H, Liu W, Wang H, Yu B, Zhang Q, et al. Enhancement of thermoelectric figure-of-merit by resonant states of aluminium doping in lead selenide. Energy \& Environmental Science. 2012;5:5246-51.

57. [57] Jones W, March NH. Theoretical solid state physics: Perfect lattices in equilibrium: Courier Corporation; 1973. 
58. [58] Ş M, Flamaropol I, Dumitru FS, Dobrescu L, Dobrescu D. Automated cooling control system through Peltier effect and high efficiency control using a DC-DC Buck converter. 2015 International Semiconductor Conference (CAS)2015. p. 281-4.

59. [59] Vázquez F, Márkus F. Size effects on heat transport in small systems: dynamical phase transition from diffusive to ballistic regime. Journal of Applied Physics. 2009;105:064915.

60. [60] Cimmelli VA. Different thermodynamic theories and different heat conduction laws. Journal of NonEquilibrium Thermodynamics. 2009;34:299-333.

61. [61] Venkatasubramanian R, Siivola E, Colpitts T, O'quinn B. Thin-film thermoelectric devices with high room-temperature figures of merit. Nature. 2001;413:597-602.

62. [62] Sumithra S, Takas NJ, Misra DK, Nolting WM, Poudeu P, Stokes KL. Enhancement in thermoelectric figure of merit in nanostructured Bi2Te3 with semimetal nanoinclusions. Advanced Energy Materials. 2011;1:1141-7.

63. [63] Zhou X, Zong P-a, Chen X, Tao J, Lin H. The structure of filled skutterudites and the local vibration behavior of the filling atom. Physica B: Condensed Matter. 2017;507:131-3.

64. [64] Rogl G, Rogl P. Skutterudites, a most promising group of thermoelectric materials. Current Opinion in Green and Sustainable Chemistry. 2017.

65. [65] Meng X, Liu Z, Cui B, Qin D, Geng H, Cai W, et al. Grain Boundary Engineering for Achieving High Thermoelectric Performance in n-Type Skutterudites. Advanced Energy Materials. 2017.

66. [66] Lu X, Morelli DT, Xia Y, Zhou F, Ozolins V, Chi H, et al. High Performance Thermoelectricity in EarthAbundant Compounds Based on Natural Mineral Tetrahedrites. Advanced Energy Materials. 2013;3:342-8.

67. [67] Huang L, Zhang Q, Yuan B, Lai X, Yan X, Ren Z. Recent progress in half-Heusler thermoelectric materials. Materials Research Bulletin. 2016;76:107-12.

68. [68] Joshi G, Dahal T, Chen S, Wang H, Shiomi J, Chen G, et al. Enhancement of thermoelectric figure-ofmerit at low temperatures by titanium substitution for hafnium in n-type half-Heuslers.

69. [69] Fu C, Bai S, Liu Y, Tang Y, Chen L, Zhao X, et al. Realizing high figure of merit in heavy-band p-type half-Heusler thermoelectric materials. Nature communications. 2015;6.

70. [70] Zhu T, Fu C, Xie H, Liu Y, Zhao X. High Efficiency Half-Heusler Thermoelectric Materials for Energy Harvesting. Advanced Energy Materials. 2015;5.

71. [71] Han C, Sun Q, Li Z, Dou SX. Thermoelectric enhancement of different kinds of metal chalcogenides. Advanced Energy Materials. 2016;6.

72. [72] Fergus JW. Oxide materials for high temperature thermoelectric energy conversion. Journal of the European Ceramic Society. 2012;32:525-40.

73. [73] Ren G, Lan J, Zeng C, Liu Y, Zhan B, Butt S, et al. High performance oxides-based thermoelectric materials. JOM. 2015;67:211-21.

74. [74] Yin Y, Tudu B, Tiwari A. Recent advances in oxide thermoelectric materials and modules. Vacuum. 2017.

75. [75] Liu Y, Zhao LD, Zhu Y, Liu Y, Li F, Yu M, et al. Synergistically Optimizing Electrical and Thermal Transport Properties of BiCuSeO via a Dual-Doping Approach. Advanced Energy Materials. 2016;6.

76. [76] Wang H, Su W, Liu J, Wang C. Recent development of n-type perovskite thermoelectrics. Journal of Materiomics. 2016;2:225-36.

77. [77] Goupil C. Continuum theory and modeling of thermoelectric elements. 2015.

78. [78] Chen G. Ballistic-diffusive heat-conduction equations. Physical Review Letters. 2001;86:2297.

79. [79] Machrafi H. An extended thermodynamic model for size-dependent thermoelectric properties at nanometric scales: Application to nanofilms, nanocomposites and thin nanocomposite films. Applied Mathematical Modelling. 2016;40:2143-60.

80. [80] Machrafi H, Lebon G. General constitutive equations of heat transport at small length scales and high frequencies with extension to mass and electrical charge transport. Applied Mathematics Letters. 2016;52:307.

81. [81] Bulusu A, Walker D. Review of electronic transport models for thermoelectric materials. Superlattices and Microstructures. 2008;44:1-36. 
82. [82] Shakouri A. Recent developments in semiconductor thermoelectric physics and materials. Annual Review of Materials Research. 2011;41:399-431.

83. [83] Rowe DM. Thermoelectrics handbook: macro to nano: CRC press; 2005.

84. [84] Zebarjadi M. Heat Management in Thermoelectric Power Generators. Scientific reports. 2016;6.

85. [85] Zhou J, Liao B, Chen G. First-principles calculations of thermal, electrical, and thermoelectric transport properties of semiconductors. Semiconductor Science and Technology. 2016;31:043001.

86. [86] Bulusu A, Walker D. Modeling of thermoelectric properties of semi-conductor thin films with quantum and scattering effects. Journal of heat transfer. 2007;129:492-9.

87. [87] Lemal S, Ghosez P, Bilc D. First principles study of heavily doped full Heusler Fe2YZ for high thermoelectric power factor. 2016.

88. [88] Chen I-J, Burke A, Svilans A, Linke H, Thelander C. Thermoelectric power factor limit of a 1D nanowire. arXiv preprint arXiv:170910511. 2017.

89. [89] Freunek M, Müller M, Ungan T, Walker W, Reindl LM. New physical model for thermoelectric generators. Journal of electronic materials. 2009;38:1214-20.

90. [90] Chen S, Ren Z. Recent progress of half-Heusler for moderate temperature thermoelectric applications. Materials today. 2013;16:387-95.

91. [91] Kim HS, Wang T, Liu W, Ren Z. Engineering Thermal Conductivity for Balancing Between Reliability and Performance of Bulk Thermoelectric Generators. Advanced Functional Materials. 2016;26:3678-86.

92. [92] Liu W, Kim HS, Jie Q, Ren Z. Importance of high power factor in thermoelectric materials for power generation application: a perspective. Scripta Materialia. 2016;111:3-9.

93. [93] Pei Y, Gibbs ZM, Gloskovskii A, Balke B, Zeier WG, Snyder GJ. Optimum carrier concentration in n-type PbTe thermoelectrics. Advanced energy materials. 2014;4.

94. [94] Dehkordi AM, Zebarjadi M, He J, Tritt TM. Thermoelectric power factor: Enhancement mechanisms and strategies for higher performance thermoelectric materials. Materials Science and Engineering: R: Reports. 2015;97:1-22.

95. [95] Zhou W, Fan Q, Zhang Q, Li K, Cai L, Gu X, et al. Ultrahigh-Power-Factor Carbon Nanotubes and an Ingenious Strategy for Thermoelectric Performance Evaluation. Small. 2016;12:3407-14.

96. [96] Suemori K, Watanabe Y, Hoshino S. Increase in thermoelectric power factor of carbon-nanotube films after addition of polystyrene. Organic Electronics. 2016;28:135-8.

97. [97] Lee W, Kang YH, Lee JY, Jang K-S, Cho SY. Improving the thermoelectric power factor of CNT/PEDOT: PSS nanocomposite films by ethylene glycol treatment. RSC Advances. 2016;6:53339-44.

98. [98] Cho C, Wallace KL, Tzeng P, Hsu JH, Yu C, Grunlan JC. Outstanding low temperature thermoelectric power factor from completely organic thin films enabled by multidimensional conjugated nanomaterials. Advanced Energy Materials. 2016;6.

99. [99] Wan C, Tian R, Kondou M, Yang R, Zong P, Koumoto K. Ultrahigh thermoelectric power factor in flexible hybrid inorganic-organic superlattice. Nature Communications. 2017;8. 